\title{
Actualidad económica y educativa. Necesidad de una vuelta a la pedagogía platónica
}

\author{
Current economic and educational situation. \\ Necessity of a return to the platonic pedagogy
}

\author{
María Isabel MEDINA MEDINA \\ Universidad Complutense de Madrid
}

Recibido: Febrero 2012

Aceptado: Abril 2012

\begin{abstract}
Resumen
Ante la perspectiva económica de la globalización, su carácter antidemocrático, el aumento de la injusticia social en el mundo que está provocando y la prioridad en educación en adaptar a los alumnos a sus exigencias, entiendo que hoy más que nunca, debemos volver a la pedagogía platónica que, en su intrínseco carácter interpersonal, sólo desea fomentar la inducción de la idea de bondad, desarrollando a su vez el sentido crítico. Esta pedagogía es el instrumento que cumpliría exactamente con las necesidades económicas y educativas actuales, para que la sociedad, lejos de ser el correlato tecnológico de la globalización, fuese quien modulase los parámetros económicos para conseguir una mayor justicia en el mundo.
\end{abstract}

Palabras clave: globalización, humanización, democracia, sentido crítico, Platón.

\begin{abstract}
In view of the prospect of economic globalization, its undemocratic nature, the increase of social injustice in the world and the priority in education to adapt students to their demands, I understand that today more than ever, we must return to the Platonic pedagogy that in its interpersonal characteristic, only wishes to encourage the induction of the idea of goodness in people waking up at the same time their critical awareness. This pedagogy could be the perfect instrument to meet today's economic and educational needs, so that society, far from being the correlative of globalization, could modulate economic parameters to achieve greater justice in the world.
\end{abstract}

Keywords: globalization, humanization, democracy, critical sense, Plato.

Nos encontramos en la era de la globalización. Una globalización que tenemos tan presente en nuestras vidas como en la consideración que somos parte indisoluble de ella. Así pues, antes de preguntarnos si realmente nos está llevando al estado de 
bienestar y felicidad que queremos, nos preocupamos por cuál es la última generación de ordenadores o de teléfono móvil para intentar consumirlos. En definitiva, damos por hecho que el consumismo de los productos de la globalización son los que nos consiguen un mayor desarrollo humano y estado de bienestar. Pero, para estar seguro de esto, deberíamos preguntarnos sobre el carácter económico que implica.

Por otro lado, están implantadas unas Competencias Educativas en el Espacio Superior Europeo que exigen una adaptación a la globalización, dificultando enormemente que los alumnos puedan plantearse concepciones distintas de desarrollo social alternativas o diferentes a la globalización planteada actualmente.

Partiendo de estas dos consideraciones, me propongo hacer un breve estudio del carácter económico de la globalización así como la actualidad educativa que impone, para plantear la necesidad de una pedagogía platónica que despierte el criterio propio y dé referencias para la concienciación de una mayor justicia social.

Así pues, en la base de la mayor diferenciación de opiniones económicas respecto al concepto de globalización, comienzo por hacer un breve estudio de sus diferentes perspectivas a fin de dar luz a la actual realidad social.

\section{Conceptos de perspectivas económicas con respecto al actual concepto de globalización}

El concepto económico planteado de la manera clásica es, según Levy-Abbad (2003), que la Globalización económica es un proceso por el cual dos o más estados estrechan sus relaciones económicas, creando mecanismos para la integración gradual de sus economías. Para ello, sus sistemas jurídicos deben alcanzar una uniformidad en la regulación del comercio exterior.

El comercio internacional es el intercambio de bienes económicos que se realiza entre los habitantes de dos o más países, de tal manera que se dé el flujo de exportaciones e importaciones entre países. Para Levy-Abbad, existe una relación de tipo estadístico entre un comercio más libre y el crecimiento económico. Indica asimismo, que todos los países, incluyendo los más pobres, tienen activos humanos, industriales, naturales, financieros que les permite producir bienes y servicios para sus mercados internos o para competir en el exterior. En resumen, la globalización económica permite que todos los países prosperen gracias a "el principio de la ventaja comparativa" porque cada país aprovechando sus activos, se concentran en lo que pueden producir mejor y después intercambiar esos productos por los productos que otros países producen mejor. Bajo estas bases económicas racionales del mercado libre está configurado nuestro actual sistema globalizador.

Continuando el análisis económico, Hoppers (2009) entiende que la comunidad internacional ha entrado en un periodo de transmisión global tremendo que ha traído la prosperidad para algunos millones de personas a través de los medios de la tecnología e Internet, pero creando más problemas sociales que soluciones. El crecimiento de la disparidad Norte-Sur en salud y acceso a los recursos coincide alarmantemente con el 
incremento en violencia, pobreza y desempleo, personas sin hogar, desplazados y la erosión de la estabilidad ambiental (Ayton-Shenker 1995, citado por Hoppers C, 2009).

Encontramos un magnífico análisis en las palabras de Castells, acerca de la relación de la globalización con la transformación de las ciudades y su correspondiente jerarquización social, bajo la hipótesis de que es el espacio el que organiza el tiempo en la sociedad. El objetivo del autor es dibujar el perfil de un nuevo proceso espacial, que se está convirtiendo en la manifestación espacial dominante del poder y la función en nuestras sociedades. Explica la organización de la economía global en los siguientes términos: "La economía internacional/global se organiza en torno a centros de mando y control, capaces de coordinar, innovar y gestionar las actividades entrecruzadas de las redes empresariales. Los servicios avanzados, incluidos finanzas, seguros, inmobiliaria, consultoría, servicios legales, publicidad, diseño, mercadotecnia, relaciones públicas, seguridad, reunión de información y gestión de sistemas de información, pero también el I+D y la innovación científica, se encuentran en el centro de todos esos procesos económicos, ya sea fabricación, agricultura, energía o servicios de diferentes clases. Todos pueden reducirse a la generación de conocimiento y los flujos de información. Estos sistemas de telecomunicaciones podrían ampliarse por todo el globo, sin embargo sólo lo hacen en las principales áreas metropolitanas del mundo. Son omnipresentes y se ubican en toda la geografía del planeta, excepto "en los agujeros negros de la marginalidad" Un ejemplo es Nueva York, Tokio y Londres en finanzas internacionales, que funcionan como una unidad en el mismo sistema de transacciones interminables. Según se desarrollan mercados emergentes se van uniendo a la red" (Castells, 1995:456)

Aplicando su teoría, Castells explica la situación económica de Madrid a partir de nuestra entrada en la Unión. Asegura que desde ese mismo momento que se consideró un mercado emergente, en esa gestión globalizadora de adquirir nuevas unidades que unan al sistema, Madrid se abrió a la inversión de capital extranjero en los mercados bursátiles, en las operaciones bancarias y en la adquisición de patrimonio empresarial y en propiedades inmobiliarias. Así en un espacio muy corto, aumentó su crecimiento económico regional. De esta forma a finales de los 80 , se incrementó la construcción de oficinas en el centro y los inmuebles residenciales de alto nivel copiaron el mismo tipo de frenesí que Nueva York y Londres. Como consecuencia, se produjo una profunda valoración del centro y un proceso de suburbanización periférica nunca antes acontecido.

Castells (1995) nos informa cómo estudios empíricos realizados por el Instituto de Desarrollo Urbano y Regional de la Universidad de California, proporcionaron en los ochenta un nuevo análisis del perfil "el nuevo espacio industrial" en que caracterizan a este espacio por separar el proceso de producción en diferentes emplazamientos mientras asegura su unidad mediante las comunicaciones. Aconseja la especificidad geográfica de cada fase del proceso de producción por la singularidad de la mano de obra que requiere en cada paso y por los diferentes rasgos sociales y medioambientales que suponen las condiciones de vida de los segmentos tan distintos de esta mano de obra. Por tanto, aquí queda demostrada la discriminación en el ámbito productivo perteneciente a este sistema globalizador, en el que quedarán los países más pobres 
afianzando cada día más su pobreza por no adaptarse a las exigencias productivas de la globalización, aumentando el número de los países que se encuentran en el agujero negro de la marginalidad.

Por tanto, se me abre el interrogante acerca de la amplitud del matiz democrático que tiene la globalización actual. Una respuesta la obtenemos en Sampedro (2002). En primer lugar expone que el mercado no es libertad, para ello sólo hace falta imaginar que alguien va a un mercado sin dinero, podrá constatar que no puede comprar nada y por tanto sin dinero, no hay libertad. Concluye "La libertad de elegir la da el dinero" (Sampedro, 2002 : 35); En segundo lugar, continua con su análisis del mercado de la globalización indicando que se encuentra bajo dos condicionantes: posibilidad instantánea de comunicaciones y transferencias económicas y por otra, amplia liberalización de las operaciones privadas y ausencia de control sobre ellas, lo que transfiere las decisiones económicas de los gobiernos con control democrático al poder privado liberado del control ciudadano. "Aunque la utilidad esencial de esta teoría consiste en legitimar el poder del dinero, se nos presenta como si tuviera las mismas virtudes democráticas del liberalismo político. Pero, la realidad es que mientras en éste último cada persona encarna un voto, en el liberalismo económico el "voto" corresponde a cada unidad monetaria y no a cada ciudadano. Por tanto, al dejar los gobiernos las manos libres al poder económico privado, los votantes han perdido el control democrático ejercido, en principio, mediante la elección de sus representantes. En otras palabras: la globalización económica es totalmente antidemocrática (Sampedro, $2002: 68$ )". Asimismo advierte de las perspectivas de la FAO respecto a la imposibilidad de lograr una justicia social en el mundo:

"Todos esos progresos no han conseguido reducir la distancia entre los países pobres y ricos, como puso de manifiesto la proclamación oficial de las Naciones Unidas para la primera década. Es más, estamos comenzando la tercera década y esa distancia ha aumentado en vez de reducirse. El hambre sigue afligiendo a subcontinentes enteros y la FAO advierte que las perspectivas no están nada claras. Según, he dicho ya, parece incluso rebasada la posibilidad de que todos los hombres puedan acceder al nivel de vida de los países más adelantados. ¡Y no digamos la humanidad de fin de siglo, aumentada casi en un 50 por ciento y teniendo a la vista un nivel adelantado muy superior al de hoy!” (Sampedro, 2009: p: 335)

Vistas estas perspectivas económicas, creo que es el momento adecuado para atender a su incidencia en la educación. Así pues, comenzaremos con la perspectiva surafricana, en un artículo en el que hace un magnífico estudio de Educación Comparada en el actual sistema globalizador, para continuar con una introducción sobre el actual planteamiento Europeo en cuanto a directrices a seguir para la Educación Superior. 


\section{La globalización y su incidencia en educación}

Atendiendo a Hoppers (2009) citando a Shiva (1997) expone que durante más de tres siglos, el reduccionismo ha gobernado como el único método y sistema científico, distorsionando la historia de Occidente al igual que la de no-occidente. Defiende un objetivo del conocimiento indígena que implica la reapropiación (Foucault, 1980, citado por Hoppers, 2009) de estos conocimientos para unir con su comprensión, desarrollo, protección y promoción. Hoppers se refiere a los sistemas de conocimiento indígenas como diferentes maneras en diferentes partes del mundo, bajo diferentes circunstancias. Suráfrica lo reconoce como conocimiento en filosofía, ciencia, tecnología, astronomía, educación, matemáticas, ingeniería, etc. Patrimonio de una nación o sociedad y mantenida por comunidades sobre países. En educación comparada e internacional, la autora indica que la imagen de la omnipotente tecnología en los últimos dos siglos ha emborronado las líneas divisorias entre los productos de ciencia moderna y la estructura formal de la racionalidad científica. Pero, incluso la ciencia a día de hoy está llamada para hacer negocio. Hace una comparativa con los objetivos de la UNESCO en cuanto a inclusividad, ciencia dialógica, debate intercultural y democrático acerca de la producción y uso del conocimiento científico y una manera de unir la ciencia moderna al patrimonio exterior de la humanidad.

Atendiendo específicamente la situación Europea, el Libro Blanco propone, entre otras cosas, la creación de un sistema Europeo de acreditación de las competencias técnicas y profesionales, apoyándose en redes Europeas de centros de investigación y de centros de fomación profesional, de empresas y de ramas profesionales. En respuesta al Libro Blanco, González indica texztualmente:

"Ya en el prólogo (Cresson y Flynn, 1996) se anteponen los cambios que introducen la información, la ciencia y la técnica en la empresa a los producidos en la escuela. Las formas de vida, el paro, las exclusión social, etc., son conceptos que se relacionan con la actividad educativa en paridad de significación. Es evidente que esta desconsideración indica que, una vez más, no se trata a la educación desde su transcendente significado sino tomándola como una actividad que prepara en función de algunas de sus utilidades menores - no por su significado como tales sino porque son naturalmente derivables de la conducta que mediatiza el conocimiento poseído- y especialmente las que se refieren al empleo, que ni tan siquiera se cita como trabajo. Importa, sobre todo anunciar que existan y ofrezcan su presencia como candidatos a los empleos que ofrece el mercado de trabajo - trabajo como mercancía- y con una preparación que responda a las exigencias laborables del producto esperado, quienes se han preparado para ello de manera prácticamente exclusiva. Es posible que personas tan cualificadas como las que elaboran este breve prólogo piensen honestamente así, pero entonces no son las más capacitadas para entender la raíz de los males que aquejan a nuestra sociedad, a los que identifican por sus nombres, en un hábito que caracteriza a un referirse a graves problemas en una sociedad que ejerce una forma débil de pensamiento; pero no es de ellos de quienes esta sociedad - del conocimiento - puede esperar soluciones para las duras dificultades que la aquejan. En este sentido si el conocimiento es efecto y acción de conocer, efecto antes en cuanto propiciador de la acción, cabría preguntarse inicialmente sobre qué se aprende en la 
sociedad del conocimiento, sin olvidar que todo aprendizaje exige de enseñanza previa. Si de lo que se trata es de dotar al individuo de conocimiento y cualidades básicas, hay que comenzar por precisar qué se entiende por ambas cosas, y esto es inexcusable en una introducción como la comentada." (González, 2001: 480-481).

Según Fejes (citado por Hodson, 2010) La Unión Europea es contradictoria en la Escuela Superior, desde que las materias son institucionalizadas, todos los principios de inclusión, movilidad, empleo, flexibilidad, habilidades transferibles están basados en el concepto del mundo globalizado. Asimismo expone que las asignaturas están hechas como vehículo de actuación y una exclusión en materia cultural son los específicos valores europeos para construir la nueva ciudadanía Europea. Basándonos en el mismo artículo de Hodson, atendemos a las palabras de Simons (2007) que incluye otro aspecto fundamental de los intereses económicos de la Unión Europea con respecto a sus cánones de imponer la educación en la base de la articulación del ciudadano europeo como "emprendedor". Ello lo realiza en la creación del Espacio Europeo de Educación Superior, dando una más alta educación de esta perspectiva como construcción de Europa como entidad visible. Así pues, orienta hacia la figura del emprendedor y la inversión para intensificar esta forma de economía como organización racional de la gobernabilidad. Esto modifica altamente las elecciones académicas para alcanzar el bienestar social propuesto por los gobiernos imperantes en la economía actual.

Ante estos planteamientos acerca de la sociedad del conocimiento que se pretende, creo fundamental investigar sobre su concepto y cronología para poder dar luz a esta idea inicial recogida en el Teeteto de Platón, que consistía básicamente en la apropiación de la ciencia para su conocimiento.

\section{Estudio cronológico del concepto de Generación de Conocimiento}

Según he podido comprobar la idea de "generación de conocimiento" está estrechamente unida al concepto de investigación y el desarrollo de nuevas ideas. De hecho, atiendo a las primeras fechas que se recogen en los estudios científicos de la base de ERIC, la primera definición la encontramos en Wisconsin Center for Education Research: "Mejorar la educación mediante la conducción de investigación básica y aplicada para llevar a cabo el desarrollo de ideas, a través de la diseminación y la demostración". Este libro en su segunda parte del título indica: veinticinco años de generación de conocimiento y mejora educativa. Klausmeier (1990).

Por lo tanto, podemos observar que ésta es la primera concepción de generación de conocimiento por llevar esta idea identificada desde mediados de los 60. Sin embargo todas las investigaciones durante la década de los 90 sobre generación de conocimiento se ha centrado en las diferentes perspectivas de ampliar, discernir y extraer conclusiones, sobre los diferentes aspectos de investigación, técnica, desarrollo...en cuanto a sociedad, economía y pedagogía que se pueden referir a las nuevas tecnologías y más específicamente a Internet. En 1999, el Banco Mundial oficializa al respecto: 
"La generación del conocimiento es muy cara, y éste es el por qué la mayor parte del conocimiento es generado en los países industriales...El Informe del Desarrollo Mundial propone que miremos los problemas del desarrollo desde una nueva formadesde la perspectiva del conocimiento...Este Informe es centrado en dos tipos de conocimiento y dos tipos de problemas que son críticos para los países en desarrollo: [i] Conocimiento sobre tecnología, el cual nosotros llamamos conocimiento técnico o simplemente know how...Típicamente, los países en desarrollo tienen menos de este know how que los países industriales, y los pobres tienen menos que los no-pobres. A estas distribuciones desiguales dentro y entre los países, nosotros llamamos brechas de conocimiento. [ii] Conocimiento sobre atributos, tales como la calidad de un producto, la diligencia de un trabajador y la credibilidad de una firma...A las dificultades representadas por el conocimiento incompleto de los atributos, nosotros llamamos problemas de información...Considerando el desarrollo desde una perspectiva del conocimiento refuerza algunas lecciones muy conocidas, tales como el valor de un régimen de libre comercio y de una educación universal...En resumen, el conocimiento provee a los pueblos de un control más grande sobre sus destinos...Sin embargo, los países en desarrollo no necesitan reinventar la rueda...En vez de re-crear el conocimiento ya existente, los países más pobres tienen la opción de adquirir y adaptar mucho conocimiento ya disponible en los países más ricos...Adquirir conocimiento implica acceder y adaptar conocimiento disponible en otras partes del mundo-por ejemplo, a través de un régimen abierto para el comercio, inversión extranjera y [licensing agreements] -y crear conocimiento localmente a través de investigación y desarrollo y de la movilización del conocimiento indígena. Absorber conocimiento implica... asegurar una educación básica universal...Comunicar conocimiento implica sacar ventaja de la nueva tecnología de la información y comunicación-a través del incremento de la competencia, provisión del sector privado y regulación apropiada- $-\mathrm{y}$ asegurando que el pobre tenga acceso...La distribución desigual de la información no puede jamás ser completamente eliminada, pero puede ser mejorada...La historia de la revolución verde muestra como la creación, difusión y uso del conocimiento puede reducir las brechas de conocimiento. También muestra que el know how es apenas una parte de lo que determina el bienestar de la sociedad. Los problemas de información conllevan a fallas del mercado e impiden la eficiencia y el crecimiento...Un desafío para los gobiernos en todas las partes es, por lo tanto, reconocer la persistencia y universalidad de las brechas de conocimiento y los problemas de información" (Banco Mundial, 1998/1999)

Mi interpretación de las palabras de El Banco Mundial es que los países pobres no tienen nada que aportar en cuanto a conocimiento y sólo adquirir aquél que han generado los países desarrollados; que esto no puede resultar extraño, si concebimos el conocimiento como mera información; y por tanto, ahí radica y sólo en este fundamento que los países pobres sean cada día más pobres. No existe una causa de poder económica. Así mismo sugiere la inclinación hacia una universalización del conocimiento que sólo quiere transformarlo en pensamiento único, que desarrollarán las empresas en un régimen de libre comercio. 
Sin lugar a dudas a equivocarme puedo comprender cómo a partir del informe del Banco Mundial, comenzamos a ver las diferentes perspectivas sobre generación de conocimiento en relación con el propio campo de las organizaciones empresariales y de la empresa privada, así por ejemplo:

La siguiente cita indica que existe cinco modos de generación de conocimiento, estos son: adquisición, destinación de recursos, fusión, adaptación y redes del conocimiento. Cuando se habla sobre generación de conocimiento se refiere tanto al conocimiento adquirido por una organización como el desarrollado en la misma. "El conocimiento adquirido no tiene por qué haber sido creado recientemente, sólo debe ser nuevo para la organización” Davenport (2001: 1).

Así pues, siguiendo una evolución cronológica, por parecerme representativa, en mi concepción que lo que llamamos Sociedad de Conocimiento ha querido apropiarse del concepto, manipulándolo para defender las nuevas tecnologías, vuelvo a González y nos encontramos con unas palabras de repulsa hacia el actual concepto y así, podemos ver en sus palabras concluyentes del artículo, la misma inquietud acerca de la posible distorsión de este concepto, que yo he detectado al comprobar que la materia relacionada con la generación de conocimiento durante toda la década de los 90 atiende exclusivamente a las nuevas tecnologías, y ya él en el 2001 concluye su artículo de esta manera:

“....Claramente, la una y la otra llamadas tecnología no sólo no son instrumentos de labor pedagógico, sino que, dejados al albur de un uso indiscriminado, irreflexivo y acrítico pueden ser netamente destructoras de un conocimiento al que no contribuyen y de unas formas de vivir derivadas de una trampa en la que la razón mal educada cae fatalmente. ¿De qué sociedad del conocimiento y de qué conocer se habla entonces? (González, 2001: 483).

También en estos años recogen esta idea, las revistas médicas preocupadas con respecto a la equidad, eficacia y eficiencia que la economía hace influir sobre la atención médica:

"La relevancia sobre el conocimiento en cuanto a la generación y reproducción del conocimiento en economía de la salud, está dada por el hecho de que en todos los países latinoamericanos, y de la mayor parte del mundo, existe aún la preocupación general de que no se están utilizando los recursos con la máxima equidad, eficacia y eficiencia en la producción de servicios de atención médica. Gran parte de los recursos asignados a la salud no se aprovechan a causa de deficientes métodos de evaluación y gestión, además del empleo de tecnologías o recursos humanos poco apropiados. En efecto la generación y reproducción del conocimiento en economía de la salud, es justamente una de las tareas para contrarrestar los efectos debido a la falta de valoraciones económicas sobre la equidad, eficacia, calidad y eficiencia, entre otros." (Arredondo y Parada, 2001: 3)

No obstante, este tipo de artículos concienciadores, explicativos y bien fundamentados acerca de la idea original de generación de conocimiento, no son tomados en cuenta en la medida de la influencia que deberían tener y nuevamente 
encontramos en los más recientes la idea relacionada con el concepto de organización empresarial, quizás cayendo en el lamentable error de ver la solución en la interiorización de las experiencias del grupo completo:

"Conjunto de conocimientos implícitos/explíctos cuya transformación y transferencia de los individuos al grupo u organización se va dando a través de lo que Nonaka y Takeuchi (1995) llaman la "espiral de los conocimientos", que tiene en cuenta la creación del conocimiento organizativo como resultado de un proceso dinámico. El punto de partida del modelo son los trabajadores individualmente y su capacidad para crear y distribuir conocimientos entre los compañeros de trabajo, donde cada individuo valora la exteriorización de los saberes. Por otro lado, interioriza las experiencias del grupo completo." (Vera, 2006: 4)

Puedo afirmar, sin lugar a dudas a equivocarme, que la evolución sufrida desde 1965 hasta el 2010, es que la idea de generación de conocimiento estaba basada en la ciencia desinteresada de cualquier otro aspecto que no fuera el desarrollo de investigación y en la creación de ideas basadas en ella, ideas para una mejor educación, pasando gracias a lo que llamamos hoy sociedad del conocimiento, al carácter más interesado económicamente que como consecuencia de los conceptos oficiales del banco mundial, y a que éstos se dirijan a un más grande desarrollo económico del mercado liberal, lleva implícito que se estudie el concepto de la idea en la organización o empresa privada.

Visto el planteamiento actual acerca de la generación de conocimiento y la introducción que he realizado con respecto a la incidencia de la globalización en la educación, creo que no debemos pasar por alto las definiciones que nos dan los expertos sobre el concepto de competencias instauradas en el Espacio Superior Europeo.

\section{Definición de Competencias para el Espacio Superior Europeo}

Centrándonos en la definición que nos otorga García (2008): viene a constatar que las competencias se plantean desde la formación para reforzar la orientación hacia la práctica o el desempeño y que el punto de referencia es el perfil profesional. Más adelante afirma que una cualificación no es más que el reconocimiento de una competencia a nivel internacional y finalmente las capacidades quedarán definidas como el conjunto de conocimientos, destrezas y aptitudes cuyo fin es la realización de actividades definidas y vinculadas a una determinada profesión. La interrelación de estas capacidades daría lugar a la competencia.

Atendiendo a Monclús (2005) viene a definir la competencia como una capacidad de una persona para realizar una determinada actividad productiva, y que la manera de determinar esa competencia, es únicamente ser capaz de realizar el trabajo de una forma segura y eficiente. 
Navío (2008) específicamente trata el diseño por competencias indicando que implica una transformación que requiere un proceso de ingeniería educativa. Según Navío, el objetivo fundamental es transformar las capacidades definidas como aptitudes o habilidades para realizar una tarea. Por tanto el diseñador de las competencias debe tener un conocimiento del escenario productivo al tiempo de un gran conocimiento de los contenidos a seleccionar para el logro de esas habilidades. Asimismo podemos ver en su obra cómo amparándose en unos mínimos de calidad, indica que debe incluir un plan de mejora permanente y que los resultados académicos entre las universidades sean buenos y puedan ser comparados con los de otros países con un mismo desarrollo económico.

Aquí, justamente, está la base de la mayor discriminación educativa que no podrá a seguir por este camino. Nunca se llegará a la justicia social en el mundo porque la implícita competitividad educativa está demostrando una enervada diferenciación de carácter económico con respecto a los países en desarrollo. Perdido cualquier atisbo de humanización en educación, atiendo con esperanza a las palabras de Barrio:

"En definitiva, para que una persona crezca como persona -en la ayuda a eso consiste el educar- para que se forme su propio criterio, necesita el estímulo adulto en la forma de una referencia de sentido que sólo puede suministrar alguien que ya lo tiene formado, por mucho que nunca se acabe de formarlo del todo (...) Sócrates nos enseñó a descubrir con su noble ejercicio mayéutico. Quien ha visto en educación un potencial revolucionario para cambiar la sociedad se ha quedado corto. El educador puede hacer mucho más que eso: humanizarla, humanizando a cada persona. Naturalmente que eso tiene un altísimo alcance social, pero sólo en la medida en que el educador con los límites de su tarea y, eso sí, conociéndolos, hace lo que puede hacer: dar referencias de sentido a través de su profesión. Hacer algo por otro es más importante que cambiar el mundo. Quizá, en el fondo, no hay otra forma de lograr un cambio en el mundo que realmente valga la pena" (Barrio, 2009: 129 y 130)

Así pues, bajo los fundamentos de que la globalización es completamente antidemocrática y que los actuales parámetros educativos están destinados a adaptar al alumno al mencionado carácter mercado liberal de la misma, dos fundamentos esenciales deben ser tenidos en cuenta en nuestra actual situación educativa: la humanización y el desarrollo del criterio propio para que desemboque en el espíritu crítico. Ambos factores forman parte de toda la obra de Platón.

Para desarrollar mejor mi perspectiva, atiendo a Masschelein, (2001) haciendo una crítica hacia los conceptos instaurados de sociedades que aprenden. Comparto la idea de que los problemas sociales o políticos no se encaminan en guiar el proceso. Estos problemas son ahora de facilitación y participación/no participación de ser activo o no activo de las órdenes dadas por parte de la economía capitalista. Se pregunta quién podría estar en contra de un aprendizaje permanente, de la calidad, de la participación de todos y aquí observa esa especie de totalitarismo blando donde la crítica real acerca del bienestar y felicidad para una parte de la humanidad se convierte en una coacción a la libertad para los que pretendemos lenguajes en educación como utopía, creación, alma, esperanza, pérdida. 


\section{La educación humanística platónica}

El concepto de educación en Platón es la ayuda a ver las cosas desde el diálogo significativo para mirar que hay detrás de lo que aparentemente se nos demuestra como verdad para continuar en el incesante camino hacia el conocimiento.

De la misma forma considero la "generación de conocimiento platónico" como la reflexión desde todos los puntos de vista posibles acerca de cualquier tema ya sea práctico o de carácter ideológico, que mediante la mayéutica o cualquier otro modo de preguntas hacen arrancar hipótesis en el educando y que por medio del diálogo guiado por el educador, se incrementan unas nuevas hipótesis, que una vez moduladas, rechazadas o aceptadas en un ciclo incremental por el propio alumno, desarrolla el espíritu crítico individual y llega a la naturaleza de las cosas por medio de la inducción para quedarse con la ideas supremas del bien, la justicia y la belleza. Por lo tanto, en ese diálogo significativo de Sócrates se encuentra el desarrollo de sentido crítico en aras de alcanzar únicamente la inducción de la idea de bondad. Si nuestro propósito es llegar a una mayor justicia social en el mundo deberíamos empezar por la enseñanza más importante:

"Pienso más bien esto último, porque has escuchado muchas veces que la enseñanza más importante es la idea de bien, gracias a la cual precisamente lo justo y lo demás que hace uso de ella se vuelve útil y provechoso... Y sabes que si no la conocemos, aunque alcanzáramos el más alto conocimiento sobre lo demás, mas no sobre ella, tal conocimiento no nos sería de ningún provecho, igual que si poseyésemos algo sin poseer el bien ¿O piensas que sirve de algo poseer cualquier cosa, pero no poseer una cosa buena? ¿O comprenderlo todo excepto el bien, sin comprender así nada bello o bueno?" (Platón, 2009 :443-444).

De este modo concebía, en la democracia de su tiempo, a los hombres de negocios, exponiendo textualmente en La República:

"Y los hombres de negocios, mientras agachan la cabeza y hacen que no los ven, clavan la lanza del dinero y hieren a quien de los demás les cede el paso en cada ocasión, crían intereses multiplicados, hijos de su padre, el capital, y crean en la ciudad mucho zángano y mucho mendigo" (Platón, 2009 : 517).

Podemos enlazar esta cita ineludiblemente con las palabras de Sampedro (2009) en la que indica que la actual civilización prefiere el "tener" al "ser" frente a los ideales humanísticos de "ser lo más con lo menos posible". Viene a constatar que los objetivos de esta tercera revolución industrial, en la que estamos inmersos, son el consumo y trabajar para el consumo ajeno. Asimismo, continúa indicando:

"Se proclama la facilidad que ofrecerá al científico la disponibilidad de terminales que pongan sobre su mesa de trabajo las más lejanas fuentes de información; pero, ¿no se contribuirá con eso, precisamente por superabundancia, a dificultar las asociaciones misteriosas de ideas que impensadamente provoca la intuición del cerebro humano? ... Un peligro bien evidente: el de la dependencia del científico, que sólo recibirá información programada por otros y que será incapaz de seleccionar independientemente" (Sampedro, 2009, p. 337) 
Por tanto en el diálogo mayéutico, en esa figura de la partera en la que el maestro hace aflorar los conocimientos en los alumnos, en base a la técnica de refutación de preguntas y respuestas, es donde Sócrates llega a la interioridad del interlocutor. De esta forma, cabe la posibilidad que en el alumno se den esas asociaciones de ideas a las que hace mención Sampedro. Sócrates profundiza siempre de manera individual sobre el pensamiento humano hasta llegar a lo que podríamos llamar hoy metacognición.

"La metacognición también se refiere a la habilidad de reflexionar desde la propia actuación. Mientras la propia-regulación puede aparecer bastante antes, la reflexión aparece más tarde en el desarrollo. Si los niños carecen de la incorporación a sus propias habilidades de aprendizaje, a duras penas podrán esperar planificar o autorregularse eficientemente". (Bransford, 2000, citado por Issitt, 2007: 390).

Esta metacognición, según Issitt (2007), apunta al régimen de verdad de Platón, y desde mi perspectiva, ya no sólo es indispensable en la formación del niño, sino que en la propia autoindagación del alumno adulto dirigida por alguien que haga pensar hacia las causas de todas las perspectivas, ineludiblemente le provocará pensar de manera ética. Estas causas comprendidas provocan una fuerte motivación que sólo puede desencadenar un mayor entendimiento de las mismas para llegar a la idea de bondad. Por otro lado, ¿no estará relacionada esta autoindagación con el concepto de inferencia, tan abandonado en nuestros días cuando se pretende una hiperespecialización y se intentan eliminar de las clases las asignaturas de carácter abstracto? Cuando habla sobre las asignaturas, da una gran importancia a la inferencia, indicando que sin ella será infructuoso cualquier tipo de conocimiento:

"Y pienso yo que la investigación de todas esas cosas que hemos expuesto, si llega hasta la asociación y el parentesco que hay entre unas y otras y deduce en qué son afines entre sí, también nos aportará algo de aquello por lo cual queremos llevar a cabo nuestro estudio, y nuestro esfuerzo no será infructuoso, mientras que en caso contrario lo será (Platón, 2009 :481)

Para intentar comprender la actualidad de los pensamientos platónicos, atendemos a Sampedro (2009) cuando afirma que tanto el sistema capitalista como el socialista aumentan con la producción creciente, por tanto todo el sistema industrial tiende a la indoctrinización interesada y esclavizante del hábito consumista o productivista. Ya Sócrates en su Apología indica sus palabras acerca de lo que acostumbraba a preguntar a sus conciudadanos:

"Mi buen amigo, siendo ateniense, de la ciudad más grande y más prestigiada en sabiduría y poder, ¿no te avergüenzas de preocuparte de cómo tendrás las mayores riquezas y la mayor fama y los mayores honores, y en cambio no te preocupas ni interesas por la inteligencia, la verdad y por cómo tu alma va a ser lo mejor posible? (Platón, 2012)

Vista la perspectiva de humanización que recoge Sócrates hacia lo que deberían ser las pautas a seguir para cualquier ciudadano del mundo actual, observamos su aplastante actualidad. Esta globalización tan preocupada por "el tener" y el productivismo, nos hace replantearnos a través de la cita, si deberíamos dirigirnos a un 
acercamiento hacia las corrientes humanísticas en ese "ser con lo menos posible". Y no sólo nos dice esto Sócrates en la medida de ciudadanos que somos sino que al tiempo nos dice que es la única manera de educar, viendo en ésta sólo una manera de cambio en hacer nuestras almas mejores. Por tanto, en esta cita podemos basar la imperiosa necesidad de su llamada "utopía" para disminuir la brecha entre los países pobres y ricos. Debemos sentir vergüenza si sólo pretendemos riquezas materiales y no nos ocupamos del enriquecimiento de nuestra alma. Un enriquecimiento del alma que se dirige hacia la idea de bondad y su inseparable justicia. Podemos fundamentar en esta cita, que sólo instaurando una educación platónica en la actualidad podríamos dirigir a esta humanidad para no tener que tildarla de esquizofrénica, según nos indica Saramago (1992) cuando compara el envío a Marte de instrumentos que estudien la composición de sus rocas y la asistencia al mismo tiempo indiferentemente a la muerte de millones de personas a causa del hambre.

\section{La educación del sentido crítico platónico. Diferencias Método Halpern y Método Socrático}

Con respecto al segundo tema de desarrollo de espíritu crítico, Leigh (2007) defiende que Platón provee a los lectores de sus diálogos de habilidades y disposiciones al igual que de afirmaciones que necesitan para evaluar críticamente un punto de vista y así, facilitar la adquisición de conocimiento para sí mismo. Añade que otra estrategia que Platón empleó fue animar al aprendiz/lector bajo qué condiciones una idea o afirmación era falsa. Por tanto, es un modelo y una aplicación de un particular tipo de pensamiento crítico en el ambiente de aprendizaje. Así pues defiende la educación platónica como el mejor camino pedagógico para hacer despertar en el alumno el sentido crítico.

A modo de ejemplo, me propongo extraer las diferencias entre un método tradicional para despertar el sentido crítico y un diseño de método socrático. El modelo que propone Halpern (1998) para enseñar pensamiento crítico se basa en estrategias para que se transfiera a través de los dominios de conocimiento y consiste en 4 partes: un componente actitudinal; instrucción y práctica de las estrategias de pensamiento crítico; actividades de enseñanza de estructura destinada a facilitar la transferencia a través de los contextos; un componente metacognitivo usado a dirigir el pensamiento. Todos estos pasos se basan en investigaciones de psicología cognitiva.

El componente actitudinal o disposicional (Halpern): Predisposición para continuar y persistir en una tarea compleja; uso habitual de planes y supresión de actividad impulsiva; Flexibilidad o abiertos de mente; predisposición a abandonar improductivas estrategias en un intento de autocorrección y un conocimiento de las realidades sociales como la necesidad de conseguir un consenso o compromiso para que los pensamientos se vuelvan acciones.

El componente actitudinal o disposicional (Método socrático): En este primer paso en el que se establecen prerrequisitos actitudinales por parte de los adultos que van a formar parte de este método, se encuentran las mayores diferencias con respecto al 
método socrático. El método socrático crea estas actitudes de manera natural a través de la reflexión y las hipótesis individualizadas planteadas por parte del maestro a los estudiantes como consecuencia del razonamiento individual de cada alumno.

Estrategias del pensamiento crítico, (Halpern): Estrategias de razonamiento verbal: incluye aquellas estrategias necesarias para comprender de defenderse contra las técnicas persuasivas que se mantienen en el lenguaje diario; Estrategias de análisis de los argumentos: en la vida real, las bases y los argumentos son complejos con razones que corren hacia la conclusión, sentadas o no sentadas en afirmaciones, información irrelevante y pasos intermedios; Estrategias para pensar como hipótesis contrastadas científicamente: la intuición de las personas como los científicos para explicar, predecir y controlar situaciones. Estas estrategias incluyen: generalización, reconocimiento de una necesidad para un gran tamaño de ejemplos, adecuadas afirmaciones y validez entre otras; Estrategias de hacer la decisión y solventar problemas: pero las que incluye son las relativas a generar y seleccionar alternativas para juzgar entre ellas. El pensamiento creativo es asumido en esta categoría por su importancia en generar alternativas y restar problemas a las metas.

Estrategias del pensamiento crítico (Método socrático): La primera gran diferencia es que no existen las estrategias verbales, éstas no son consideradas en el diálogo socrático, desde que el principio fundamental por el que se rige es en la estrategia de pensamiento. El maestro entenderá qué quiere decir el alumno y mediante las diferentes alternativas hipotéticas, le hará clarificar y dar exactitud a sus pensamientos a través del lenguaje. El lenguaje sólo es un medio, nunca un fin; La siguiente diferencia estriba en las estrategias para analizar pensamientos de la vida real. Por el contrario, el diálogo socrático se basa en la conceptualización de ideas de diálogos de envergadura humana.

Enseñanza de la estructura para promover la transferencia (Halpner): Dibujar un diagrama que organice la información; ¿Qué información adicional necesitas antes de responder la pregunta?; Explica por qué seleccionas una particular alternativa del multiple-choice. ¿Qué alternativa es la segunda mejor? ¿Por qué?; asienta el problema al menos en dos vías; ¿qué información es más importante? ¿qué información es menos importante? ¿por qué?; haz una lista con dos soluciones al problema; ¿qué es incorrecto de la información que se da en la pregunta?; presenta dos razones que apoyen la conclusión y dos razones que no la apoyen; identifica el tipo de técnica persuasiva que es usada en la pregunta; ¿qué dos acciones podrían llevar a mejorar el diseño del estudio que se ha hecho?.

Enseñanza de la estructura para promover la transferencia (Método socrático): En primer lugar, nunca se realizaría ningún tipo de diagrama que organice la información, la información que aporta en un primer paso, el método socrático cuenta, en algunos casos, con expertos en filosofía que han reflejado su pensamiento personal acerca de los distintos sentidos sobre la justicia, la virtud, el conocimiento, etc. Por tanto, cada matiz de pensamiento de cada alumno, es tenido en cuenta para su posterior valoración y todos ellos son válidos si se acercan al conocimiento racional de la bondad; En segundo lugar, el maestro nunca pregunta qué más necesitas para contestar, sino que a 
través de su hipótesis hará que el alumno descubra por sí mismo la información de la que carecía; En tercer lugar, nunca habrá un multiple-choice al que restringir el pensamiento, el pensamiento será siempre individual, la libertad de pensamiento es la base del diálogo socrático; Por último, nunca será incorrecta la pregunta del maestro y mucho menos utilizará técnicas persuasivas, dado que se basa en el diálogo moral que establece que nadie busca influenciar, seducir o manipular al otro. Por el contrario todas las preguntas sirven de ayuda para la conceptualización de las ideas que puedan ser importantes para la vida en la capacidad de la inducción.

Componente metacognitivo usado a dirigir el pensamiento (Halpner): La actividad explicativa de este componente la basa en dar a sus alumnos las características que determinan los horóscopos de la personalidad de los individuos y que razonadamente comparen su propia personalidad con la argumentada por éstos.

Componente metacognitivo usado a dirigir el pensamiento (Método socrático): El componente metacognitivo usado a dirigir el pensamiento es racional y moral: "Y juzga teniendo en cuenta lo siguiente: lo que se atiene a lo que es siempre igual, es inmortal y es la verdad, siendo así él mismo y produciéndose en lo que es semejante a él” (Platón, 2009 : 567). Este componente es usado para los casos fácticos de la vida real, y supone nuestro pensamiento crítico individual sin dejar de ser justos y buenos al utilizarlo.

\section{Conclusiones}

La presente investigación ha tenido como objetivo hacer un análisis de la actual situación de la globalización, que está incidiendo en los nuevos parámetros educativos y consecuentemente en la futura sociedad para demostrar la necesidad de una vuelta a la pedagogía platónica.

Platón a través del pensamiento que suscita todo conocimiento de cada materia, orden, sujeto u objeto orienta su reflexión desde cada perspectiva y plantea las preguntas a fin de que otra persona pueda dar respuesta bajo su individual prisma y a partir de ahí suscitar nuevas preguntas que sólo cada ser humano en base a su razón absolutamente personal puede expresar, intentando llegar a esa aproximación con respecto a la verdad, sin abandonar nunca la perspectiva ética de cada referencia expresada.

Por tanto, Platón ya planteaba una escuela inclusiva desde el momento que lo importante, para él, era la persona a la que se dirigía, el más absoluto respeto a la individualidad e interioridad del interlocutor con respecto a educación. La eficacia de esta escuela inclusiva que creemos haber descubierto apenas hace unos años, ya estaba demostrada por Platón en todas las estrategias de pensamiento dirigidas a la educación.

Así pues, existen dos motivos que se correlacionan con respecto a la necesidad acuciante de la pedagogía platónica en nuestros días. En primer lugar y en la base de que la educación platónica se fundamenta en un siempre inacabado conocimiento para 
llegar a ser mejores personas, encontramos su independencia educativa, que erradicaría los marcados parámetros educativos determinados por la economía, que están confundiendo la acción y el efecto de la educación. La educación actual basa sus objetivos en unos determinados efectos económicos prácticos, lejos de la acción platónica que pretende los efectos de crecimiento personal, que consecuentemente desembocarían en los prácticos, ya como consecuencia o de segundo orden en los económicos, rechazando aquellos que no sean afines a la bondad, por tanto en aras de la economía marcada por la justicia social en el mundo.

En segundo lugar, la pedagogía platónica, se basa fundamentalmente en el desarrollo del criterio propio, en la base del uso de la racionalidad completamente individual del ser humano, por tanto ya tenemos el primer paso para el desarrollo del espíritu crítico. Este desarrollo de espíritu crítico permitiría por un lado, que se entorpeciera el incremento de las disposiciones actuales hacia la universalización de un determinado conocimiento previamente establecido por la economía imperante; y por otro lado, facilitaría una revolución ideológica para cambiar los parámetros de economía sobre educación -que irremisiblemente desemboca en la configuración de la sociedad- y se dirijan por el contrario, a que sea la sociedad la que module la economía imperante en la actualidad.

Por tanto y a modo de conclusión final, desde mi perspectiva que entiende la educación como una intervención intencional para hacer hombres libres y responsables y desde la base de que es la sociedad la que debe modular los parámetros económicos y que lejos de ello, éstos están poniendo todo su esfuerzo en dirigir la educación hacia una apropiación de la misma para promover sus ideales y sacar de los individuos una rentabilidad económica que beneficia a una sola parte de la humanidad, considero de necesaria urgencia una vuelta a la pedagogía platónica para desarrollar el criterio propio del alumnado y el amor al conocimiento humanístico que desembocaría, una vez llegado a la idea suprema de la bondad, en una mayor justicia social en el mundo.

\section{Referencias bibliográficas}

ARREDONDO, A y PARADA, T. (2001). Tendencias de la generación y de conocimiento sobre evaluación económica y salud. Rev. Méd. Chile. V $129 n 8$. Santiago. http://www.scielo.br/scielo (Consultado el 5.X.2011)

BANCO MUNDIAL. (1998-1999) World Development Report: Knowledge for Development. Overview, p. 1-14.

BARRIO, J. M. (2009). El balcón de Sócrates Una propuesta frente al nihilismo. Madrid. Rialp, S.A.

DAVENPORT (2001). http://redalyc.uaemex.mx/redalyc/pdf/904/90411003.pdf (consultado el 7.V.2011)

GARCÍA, J. A. (2008) Las competencias y su implicación conceptual: un reto para el espacio europeo de educación superior. Madrid. Davinci Continental. S.L. 
CASTELLS, M. (1995) La Era de la Información. México. Siglo XXI.

GONZÁLEZ, F (2001). Generación del conocimiento y actividad educativa. Revista Complutense de Educación 480 Vol. 12 Núm. 2, 427-484

HALPERN, F. (1998) Teaching Critical Thinking for Transfer Across Domains. Dispositions, Skills, Structure Training, and Metacognitive Monitoring. American Psychological Association Vol. 53. N. 4. 449-455.

HODGSON, N. (2010). What does it mean to be an educated person? Journal of Philosophy of Education. Vol. 44. No. 1.

HOPPERS, C. (2009) Education, Culture and Society in a Globalizing world. Implications for Comparative and International Education. A Journal of Comparative and International Education. V39 N5 P601-614

ISSITT, J (2007). Evidence and Metacognition in the New Regime of Truth: Figures of the Autonomous Learner on the Walls of Plato's Cave. Journal of Philosophy of Education, Vol. 41, No. 3,

KLAUSMEIER, J. (1990). The Wisconsin Center for Education Research. TwentyFive Years of Knowledge Generation and Educational Improvement. 020 Collected Works: General. ERIC, Resources in Education (RIE)

LEIGH, F (2007). Platonic Dialogue, Maieutic Method and Critical Thinking. Journal of Philosophy of Education; v41 n3 p309-323.

LEVY- DABBAH, S (2003) La Globalización Económica. Cómo manejar con éxito los negocios entre México y Sudamérica. México, ISEF.

MONCLÚS, A. (2005). La perspectiva de la educación actual. Editorial Salamanca. Témpora. España.

MASSCHELEIN, J. (2001). The Discourse of the Learning Society and the Loss of Chilhood. Journal of Philosophy of Education, Vol. 35, No.

NAVÍO, A. (2008). Un nuevo modelo de Formación para el siglo XXI: La Enseñanza Basada en Competencias. Competencia Profesional y Formación Basada en Competencias: Apuntes para la Educación Superior. Editorial Barcelona. Davinci.

PLATÓN (2009) La República. Madrid. Akal.

PLATÓN (2012) La Apología. http://www.academiasocrates.com/socrates/apologia.php.

SAMPEDRO, J.L. (2002) El Mercado y la globalización. Barcelona. Destino.

SAMPEDRO, J.L. (2009) Economía Humanista. Algo más que cifras. Barcelona. Random House Mondadori.

SARAMAGO, J. (1992). Todos los Nombres. Editorial Alfaguara. Madrid

VERA, G. (2006). La Generación de conocimiento organizacional como factor clave en el desarrollo de la capacidad de innovación: el caso de una empresa textil 
poblana. Congreso Iberoamericano de Ciencia, Tecnología, Sociedad e Innovación CTS + I. Palacio de Minería. Junio. Mesa 12. http://www.oei.es/memoriasctsi/mesa12/m12p29.pdf (consultado el 5.X.2010)

\section{Correspondencia con la autora:}

$\mathrm{M}^{\mathrm{a}}$ Isabel MEDINA MEDINA

Dirección: Estafeta, $11-3^{\circ}$ B. 28041 Madrid

Email: madagascar.200@gmail.com 